\title{
Minimally Invasive Widening of the Facet Joints in Cervical Radiculopathy by Modified Needles: Technical Report
}

GeonMok Lee ${ }^{1}$, HyangJoo Lee ${ }^{2}$, Yong Suk Kim³ ${ }^{3}$ JongHyun Han ${ }^{4}$, EunYong Lee ${ }^{5}$, HoSueb Song ${ }^{6}$, TaeHan Yook ${ }^{7}$, JaeSoo Kim ${ }^{8}$, KyongHa Cho ${ }^{1}$, SeRin Kang ${ }^{1}$ and SangHoon Yoon ${ }^{1}$

1. Department of Acupotomy, LeeGeonmok Wonli Korean Medicine Hospital, Seoul 137-829, Republic of Korea

2. Department of Anesthesiology, LeeGeonmok Wonli Korean Medicine Hospital, Seoul 137-829, Republic of Korea

3. Department of Acupuncture and Moxibustion, Kyunghee University, Seoul 135-501, Republic of Korea

4. Department of Pharmacology, Wonkwang University Iksan 570-749, Republic of Korea

5. Department of Acupuncture and Moxibustion, Semyung University, Chungju 380-080, Republic of Korea

6. Department of Acupuncture and Moxibustion, Gachon University, Seongnam 461-701, Republic of Korea

7. Department of Acupuncture and Moxibustion, Woosuk University, Wanju 565-701, Republic of Korea

8. Department of Acupuncture and Moxibustion, Daegu Hanny University, Daegu 706-828, Republic of Korea

\begin{abstract}
Surgical treatment and ESI (epidural steroid injection) are widely used forms of treatment for cervical radiculopathy but they are controversial and burdensome for patients. To relief pain fast without side effects, we devised a new minimally invasive treatment method that widens the facet joints to decompress nerve roots and release the muscle spasm in cervical radiculopathy with acupuncture needles with blunt tip and mini-scalpel, and named it modified acupuncture procedure. MAP (Modified acupuncture procedure) was administered for 37 patients (mean age $=53.1$ years, follow-up $=14.2$ months) with cervical radiculopathy who did not recover from 4 weeks of nonsurgical treatment. We analyzed clinical outcomes of patients before and after the procedure through VAS (Visual Analogue Scale) and NDI (Neck Disability Index). On average, patients received 1.4 MAP (modified acupuncture procedures). The VAS score difference on the day after procedure and at 1 year follow-up was $36.8 \pm 26.5$ (from $60.1 \pm 25.3$ at the baseline to $25.3 \pm 17.8$ at the reading $)(P<0.01)$ and $31.0 \pm 30.4(29.0 \pm 21.8$ at the reading) respectively. The NDI value dropped by $19.9 \pm 18.3$ (from $37.2 \pm 19.7$ at the baseline to $17.2 \pm 15.0$ at the reading) $(P<0.01)$ on 1 year follow up. MAP was found to have clinical efficacy for cervical radiculopathy.
\end{abstract}

Key words: Minimally invasive procedure, facet joint, cervical radiculopathy, acupuncture, acupotomy, adhesion, muscle spasm.

\section{Introduction}

Symptoms of cervical radiculopathy include pain in the arms, neck or shoulder blades, paresthesias, numbness and sensory changes, weakness, and abnormal deep tendon reflexes [1]. Cervical radiculopathy is a relatively common ailment that accompanies severe pain, and its morbidity rate is reportedly about $0.3 \%$ [2]. The most common cause of

Corresponding author: GeonMok Lee, Ph.D., president of association, research field: acupuncture. E-mail: geonmokbo@gmail.com. cervical radiculopathy $(70 \%-75 \%)$ is foraminal encroachment of the spinal nerve resulting from complex factors such as reduced disc height and degenerative changes in uncovertebral joints and facet joints. Herniation of the nucleus pulposus is responsible for the remaining $20 \%$ to $25 \%$ of cervical radiculopathy [3].

Among different types of treatment for cervical radiculopathy, one conservative treatment involves ESI [4]. About $60 \%$ of the patients treated with ESI have reported positive long-term relief [5], while some 
studies report improvement of symptoms only in $16-17 \%$ of the patients [6]. However, serious neurological side effects like loss of vision, stroke, paralysis, and death are reported after ESI [7], and this treatment alone is not considered sufficient as an alternative to surgery [8].

About $30 \%$ of the patients who received this treatment and still have pain decide to undergo surgery [9]. However, some suffer from complications following surgery and require additional surgery due to severe pain. According to the latest research, surgery resulted in the same outcomes as those of physical therapy without surgery after two years in terms NDI and arm pain [10].

If pain persists even after ESI, both doctors and patients find themselves at a loss because there is no remedy. Since these are the patients who can no longer endure pain and decide to undergo surgery with fear. So a safe treatment that offers quick relief, which is maintained in the long term is necessary. To ensure fast pain relief with less side effects, it is crucial to quickly address high pressure on nerve roots.

A knife shaped acupuncture KA (knife acupuncture) was used as a tool to release synechia. By combining the KA with BTA (blunt tip acupuncture), we devised a new treatment procedure to treat foraminal encroachment of the spinal. KA is used to widen the facet joint to decompress the intervertebral foramina and the spinal canal without open surgery and BTA is used to release the muscle spasm. We named this the "MAP (modified acupuncture procedure)". Here we report clinical efficacy and valid results without major adverse effects.

\section{Materials and Methods}

\subsection{Inclusion Criteria}

Neck pain, arm pain, and/or neurological symptoms.

Not relieved for at least 4 weeks by nonsurgical treatments (exercise, analgesics and ESI).
Diagnosed cervical disc causing nerve root compression by MRI.

\subsection{Exclusion Criteria}

Underwent surgery in the cervical area.

Symptoms of myelopathy, and/or fibromyalgia.

\subsection{Method of Evaluation}

VAS (visual analog scale) and NDI (neck disability index) of individual patients were measured prior to the procedure and one year after the procedure via phone. Findings were analyzed by using SPSS $18.0 \mathrm{~K}$ for Windows and a paired sample t-test was performed to determine the differences between NDI and VAS before and after the procedure.

\subsection{Subjects}

The study protocol was approved by the IRB (institutional review board) of Semyung University Hospital (Chungju, South Korea) with an assigned number of 1412-06. VAS and NDI scores were assessed for 39 individuals during the research period. Among these were excluded, one patient who underwent surgery and one patient with fibromyalgia. The average age of the remaining 37 patients (15 male and 22 female) is 53.1. The patients received MAP about 1.4 times each, with maximum of three times. The patients' initial mean NDI was 37.2 and their mean VAS was 60.1. Pain had lasted 11 months on average and follow-up procedures were carried out for 14 months (Table 1 ).

Table 1 Demographic characteristics.

\begin{tabular}{ll}
\hline Demographic characteristics & \\
\hline Patients (N) & 37 \\
Age (years) & $53.1 \pm 9.6$ \\
Sex (M/F) & $15 / 22$ \\
Duration of pain (months) & $11 \pm 6.2$ \\
Neck disability index (\%) & $37.2 \pm 19.7$ \\
Visual analogue scale & $60.1 \pm 25.3$ \\
Period of F/U (months) & $14.2 \pm 3.3$ \\
Frequency of procedure & $1.4 \pm 0.64$ \\
\hline
\end{tabular}




\subsection{Study Intervention}

The MAP is devised as a complex treatment procedure involving two stages. Prior to the procedure, the dermatomal distribution that a patient complains of pain is compared to the area of damage shown in MRI, in order to determine the location of procedure. The provider then checks tender points by feeling the facet joints of the patient, and confirms the area for the procedure, taking into account the patient's MRI and symptoms.

\subsection{Procedure 1: KAP (knife acupuncture procedure)}

The patient lies on the operational table in prone position and takes a position that bends the cervical vertebrae as much as possible. For the procedure, the KA1, made by Dongbang Acupuncture Needle Company was used with $1 \mathrm{~mm}$ diameter, $80 \mathrm{~mm}$ needle length, and a flat blade of $1.0 \mathrm{~mm}$ at the tip (Fig. 1a). After MRI and X-ray examination, the area for the procedure is determined by correlating MRI findings and the patient's symptoms. The selected area is marked with a surgical marking pen to show where the KA1 should be inserted. The KA1 insertion point for a herniated cervical disc is a facet (zygapophyseal) joint of the cervical vertebrae. The facet joint is located 13-23 mm (depending on individual patients' characteristics) away from the middle point between the spinous process on both sides. The facet joint is first felt with the hand, marked, or confirmed through $\mathrm{C}$-arm. During the procedure, an oxygen line is placed into the patient's nose for easier breathing and the patient was asked to bend his or her neck to ensure maximum exposure of the neck bone. Local anesthesia and/or intravenous anesthesia (midazolam) was applied.

The end blade of the KA1 is held parallel to the spine in the longitudinal axis and perpendicular to the skin upon insertion into the facet joint in the cervical vertebrae. The facet joint section is $10 \mathrm{~mm}$ wide and 5-7 $\mathrm{mm}$ long from front to back, and its angle in the neutral position of the neck upon standing is tilted $45^{\circ}$ against the ground. The KA1 is injected through the skin and made to reach the bone surface of the facet joint, and then its blade tip is made to spin $90^{\circ}$. So the needle is parallel to the facet joint. The KA1 is moved alongside the facet joint surface to incise articular capsules of the cervical joints and is injected about 3 $\mathrm{mm}$ into the capsule to ensure that it becomes fixed between the joints, and then is shaken up and down and sideways to separate the joints. The same procedure is repeated until the provider can feel that the joints are completely detached, and then the KA1 is removed. Pressure is applied for three minutes to stop bleeding (Fig. 2).

\subsection{Procedure 2: BTAP (blunt tip acupuncture procedure)}

The KA 2 is $1 \mathrm{~mm}$ in diameter, $100 \mathrm{~mm}$ in overall length, $70 \mathrm{~mm}$ in length of the needle, and $30 \mathrm{~mm}$ in length of handle. The tip is a flat blade with 0.8-1.0 mm length (Fig. 1b). The BTA is " $Z$ " shaped, with a handle $100 \mathrm{~mm}$ in length, a needle $110 \mathrm{~mm}$ in length,
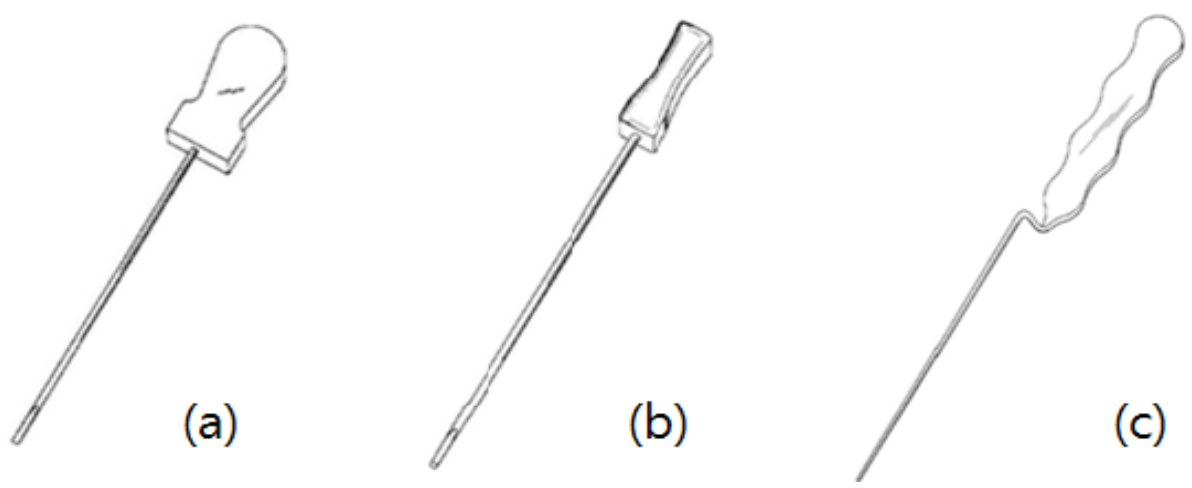
Fig. 1 Modified acupuncture needles.

(a) Knife acupuncture 1-KA1, (b) Knife acupuncture 2-KA2, (c) Blunt tip acupuncture-BTA.

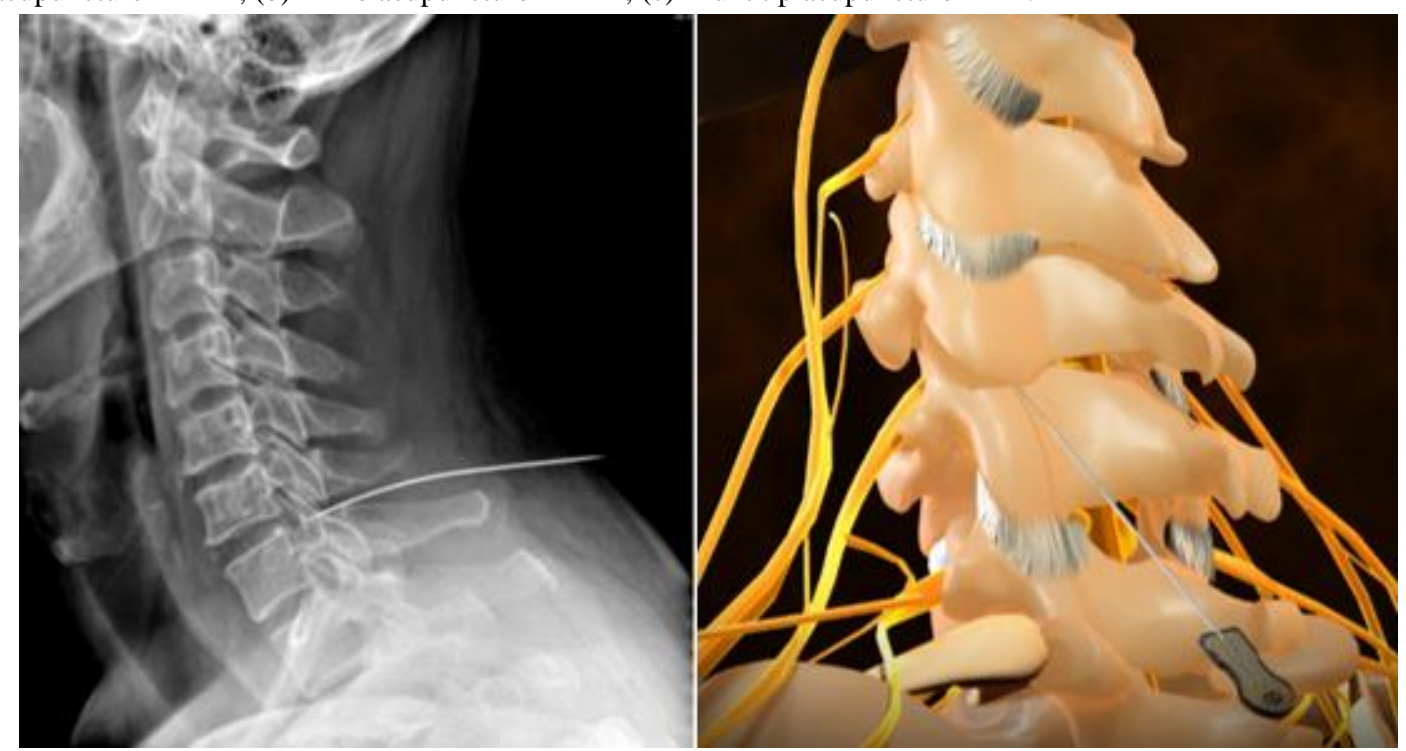

Fig. 2 Image of knife acupuncture procedure model.

and a round tip $1 \mathrm{~mm}$ in diameter (Fig. 1c). As in procedure 1 , the patient lies on the operation table in prone position and takes a position that makes the cervical vertebrae as bent as possible.

The apex of the spinous process of the C7 (7th cervical) vertebra is perceived with the hand and marked. After marking the area for KA2 insertion with a surgical marking pen, both the KA2 and BTA needle are used for the procedure. First, the epidermis of the area is pierced using the KA2. The provider holds the KA2 in his or her hand and positions the needle perpendicular to the skin and the flat blade in the same direction as the longitudinal axis of the human body. The KA2 is inserted $3-5 \mathrm{~mm}$ into the epidermis in a swift motion and then is removed immediately. Afterwards, the provider holds the BTA needle in his or her hand, and inserts the needle 3-5 $\mathrm{mm}$ into the epidermis perpendicularly, directs the needle tip at the external occipital protuberance, and then inserts the needle all the way to the end of the transvers process of the 1 st cervical vertebra (Fig. 3a). In doing so, the synechia of the fascia is removed, and after two to three iterations of relaxation treatment, the BTA needle is retracted to the epidermis from which it was first inserted. The needle tip is then spun $30^{\circ}$ so that the needle can enter laterally in order to detach the synechia all the way to lateral surrounding tissue of the trapezius muscle to release the trigger points of muscle spasm (Fig. 3b). The angle of the needle tip is varied sufficiently with the inserted point, thereby extensively relaxing the synechia between the skin and muscles.

\section{Results and Discussion}

\subsection{Clinical Outcome}

When corrected as a $100 \%$ unit, the average NDI after the procedure was 19.9, while VAS decreased by 31.0 on average, a significant outcome NDI improvement was seen in 21 cases at $56.8 \%$, while 20 cases had VAS improvement of $54.1 \%$. There was no significant difference $(P<0.01)$ between male and female patients in terms of the procedure's effects. There were no adverse effects (Table 2, Fig. 4).

Cervical radiculopathy pain has been thought to be caused not only by root compression but also by instability caused by disc degeneration or facet joint osteoarthritis because it usually precedes radicular symptoms in the arms/fingers [11]. Herniated cervical 
Modified Needles: Technical Report

disc is regarded as the main cause of neck pain and radiating pain [3]. Cervical disc disease is not confined to a single disease but is rather an

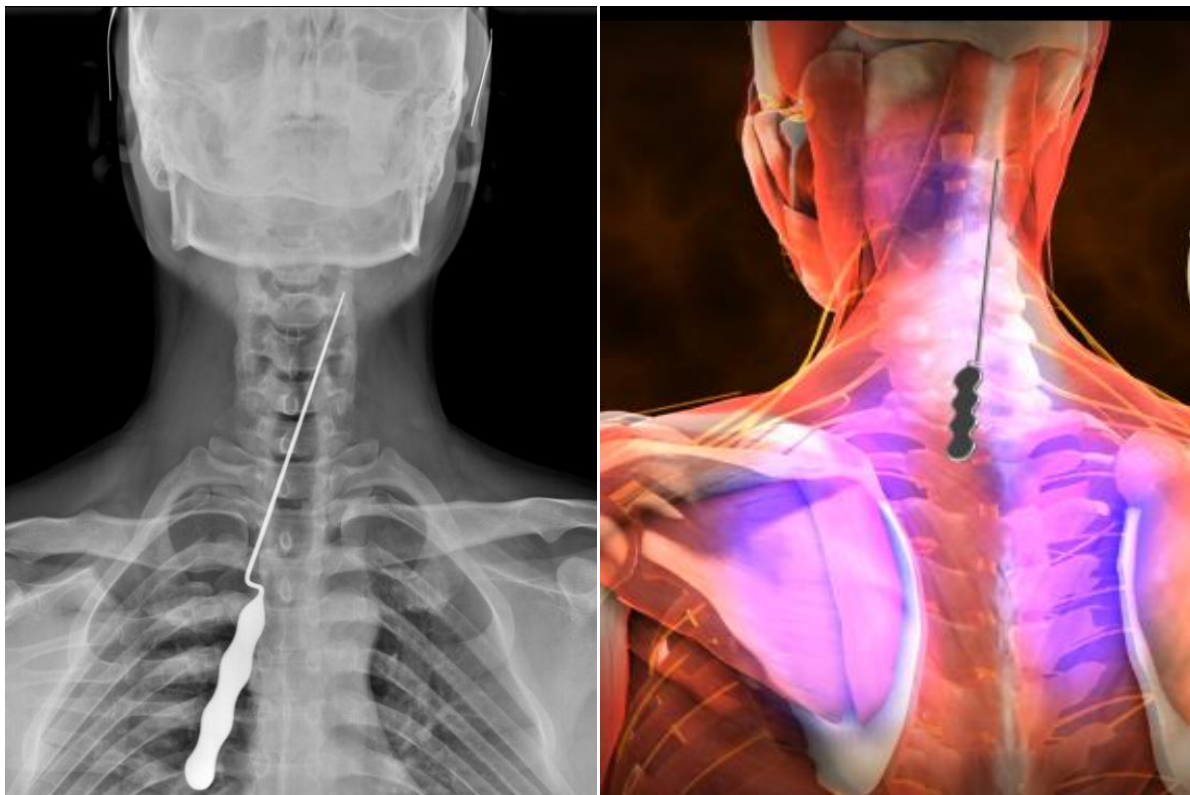

(a) BTAP to the transverse process

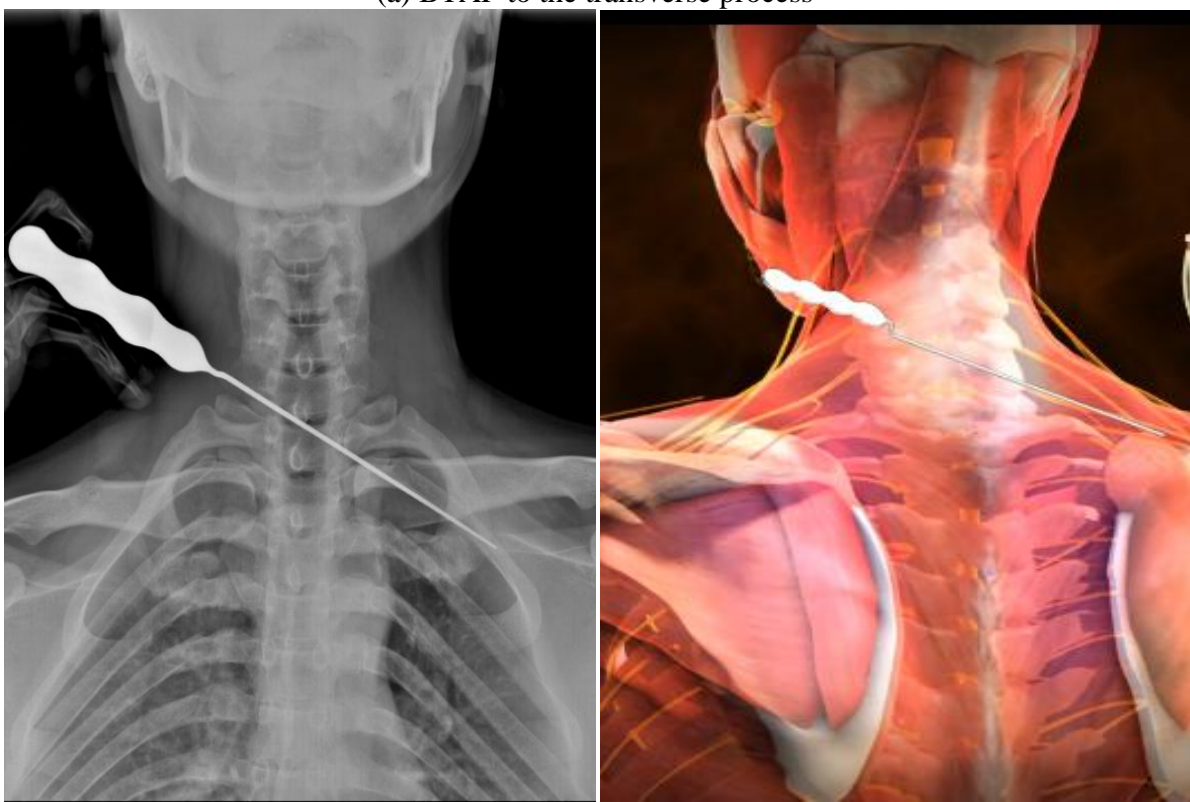

(b) BTAP to the trapezius muscle

Fig. 3 Image of blunt tip acupuncture procedure model.

Table 2 Pre-treatment and post-treatment comparison.

\begin{tabular}{llll}
\hline Pre- and Post-treatment comparison & & & \\
\hline NDI & Pre-treatment & \multicolumn{1}{l}{1 year F/U } & \\
\hline NDI score & $37.2 \pm 19.7$ & & \\
Average relief of NDI & $19.9 \pm 18.3$ & & \\
$\geqq 50 \%$ improvement NDI & $21 / 37(56.8 \%)$ & & 15.0 \\
\hline VAS & Pre-treatment & 1 day after-treatment & $29.0 \pm 21.8$ \\
\hline VAS score & $60.1 \pm 25.3$ & $25.3 \pm 17.8$ & \\
\hline
\end{tabular}




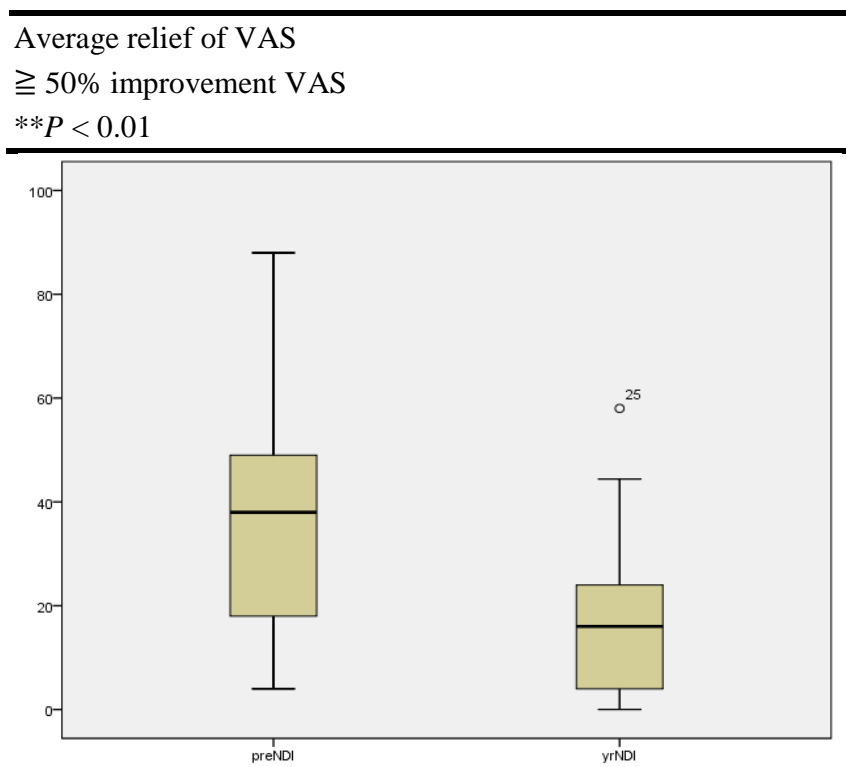
$36.84 \pm 26.5$
$31.0 \pm 30.4$
$22 / 37(59.5 \%)$
$20 / 37(54.1 \%)$

$* * P<0.01$

Fig. 4 Coefficient of quartile deviation of pre and post NDI, VAS.

all degenerative changes occurring on the cervical vertebrae, and the most common degenerative changes include herniated cervical disc, formation of osteophytes, and hypertrophy of the ligamentum flavum, which clinically cause cervical pain, nerve root disease, and myelopathy [12].

In general, about $80 \%$ of the patients complaining of cervical pain respond to conservative treatment [13], and thus nonsurgical treatment must precede. The effects of ESI, often used as conservative treatment, are disputable. Even its safety is questionable as it may lead to such side effects as severe neurologic sequelae from the spinal cord and brainstem infarction. Thus it must be performed with caution [14].

Of patients who complain of severe pain caused by cervical radiculopathy, $26 \%$ decide to undergo a surgical operation in less than three months after diagnosis, due to radicular pain, sensory loss, and muscle weakness [15]. For cervical radiculopathy, ACDF (anterior cervical decompression and fusion) is the most common surgical procedure. However, while ADCF is effective in controlling extreme pain initially, it showed no difference after two years in terms of pain control and functionality compared to conservative treatment [16]. Moreover, once ACDF is performed, the pressure on the adjacent discs is increased upon bending, which tends to further accelerate degenerative changes in the cervical vertebrae [17].

Even though patients want fast pain relief, they are hesitant to make a decision on a surgical operation due to fear of the procedure itself and of adverse effects. Considering this, we proposed a new treatment that combines KA and BTA to ensure fast and relatively persistent relief and safety.

The use of KA and BTA on lumbar spinal stenosis was reported before [18]. But this is the first research of KA and BTA on patients with cervical radiculopathy. As mentioned above, treatment is mainly performed on facet joints. We reduce the elevated pressure within the spinal canal space and intervertebral foramina by widening the facet joints that were narrowed with hypertrophy caused by osteoarthritis. Thus, radicular pain is reduced simply by decompressing nerves in chronic phase.

Afterwards, a long BTA needle is used to release the muscles to ease overall tension on the cervical vertebrae and to treat synechia and cicatrix of fascia, thereby addressing the problem of straightening. The principle of this treatment is to recover this balance by releasing the extensive synechia with tensed muscle at the back of the cervical vertebrae. By this procedure, 
the damaged muscles and fascia are treated, and cicatrix and synechia are released, allowing mere active blood circulation and helping the muscles to recover to their normal state.

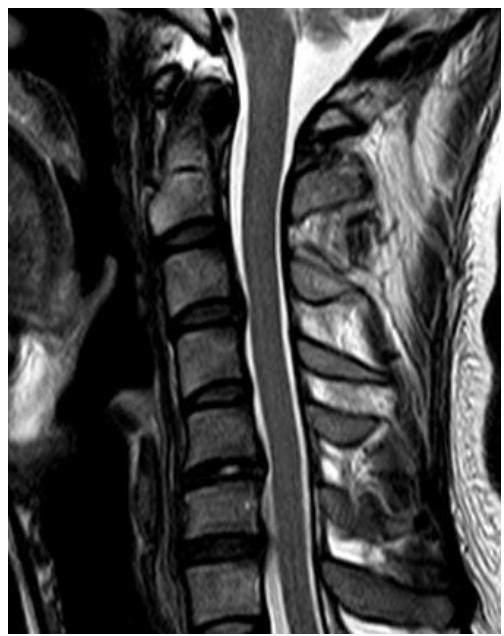

(a) MRI image before MAP

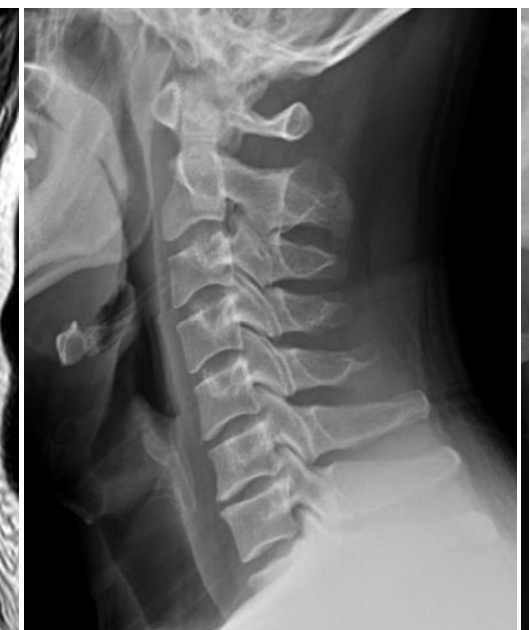

(b) X-ray image before MAP

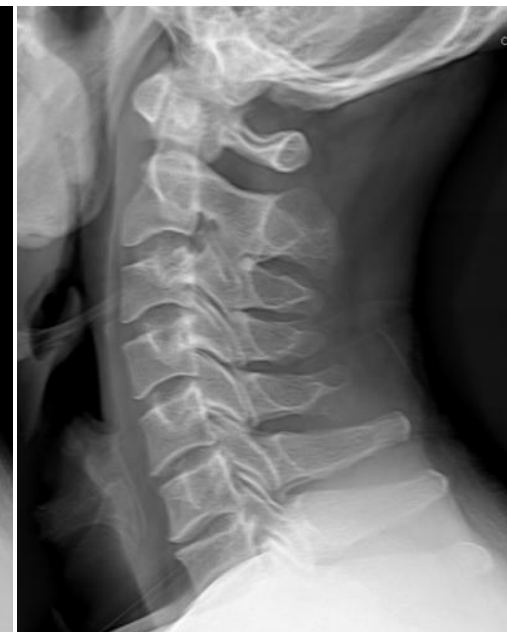

(c) X-ray image after MAP

Fig. 5 Restoration of cervical lordosis (before and after image of modified acupuncture procedure after 1 month).

in this study. In the case of acute cervical disc herniation, VAS scores dropped quickly after each weekly procedure, and conditions improved after about 4-6 weeks. This leads us to believe MAP to be an alternative to treating even an acute herniated cervical disc condition over time. Further research is required for acute pain in the cervical radiculopathy.

In general, the improvement was quick and a pain-free state was maintained for a long duration even after discontinuing further procedures as they no longer needed it. Additional outcomes of this treatment include disappearance of headaches, brighter vision, neck comfort, better digestion, and relief of shoulder pain. In some cases we find restoration of cervical lordosis on radiographs (Fig. 5).

\section{Conclusions}

MAP were done to the patients who had not relieved at least 4 weeks by nonsurgical treatments (exercise, analgesics and ESI), advised to have the operation. The method of the MAP is that the facet joints are separated without removing the bones and the discs, and then the nerve root is decompressed.
The MAP is done average 1.4 times, and the patient had the three weeks to the four weeks of a period of time between the 1st and the 2nd operation/procedure. As a result of a follow-up for average 14 months, VAS and NDI improved, and the imoroved condition showed similiar result continuously. These patients could enjoy the daily life and exercise after one year of the procedure. As the result tells, chronic cervical radiculopathy can be treated by the procedure of facet joint separation. So, in the method of decompression of nerve root, this procedure will be another alternative.

\section{Acknowledgments}

I am indebted to Dr. Hee-Choon S. Lee for his critical review.

\section{References}

[1] Bono, C. M., Ghiselli, G., Gilbert, T. J., Kreiner, D. S., Reitman, C., Summers, J. T., Baisden, J. L., Easa, J., Fernand, R., Lamer, T., Matz, P. G., Mazanec, D. J., Resnick, D. K., Shaffer, W. O., Sharma, A. K., Timmons, R. B., Toton, J. F., and North American Spine Society. 2011. "An Evidence-Based Clinical Guideline for the Diagnosis and Treatment of Cervical Radiculopathy from 
Degenerative Disorders." The Spine Journal 11 (1): 64-72.

[2] Salemi, G., Savettieri, G., Meneghini, F., Di-Benedetto, M. E., Ragonese, P., Morgante, L., Reggio, A., Patti, F., Grigoletto, F., and Di-Perri, R. 1996. "Prevalence of Cervical Spondylotic Radiculopathy: A Door to Door Survey in a Sicilian Municipality." Acta. neurologica Scandinavica 93 (2-3): 184-8.

[3] Cervical-Radiculopathy, S. C., and Michael, G. F. 2005. "Cervical Radiculopathy." New England Journal of Medicine 353 (4): 392-9.

[4] Benyamin, R. M., Singh, V., Parr, A. T., Conn, A., Diwan, S., and Abdi, S. 2009. "Systematic Review of the Effectiveness of Cervical Epidurals in the Management of Chronic Neck Pain.” Pain Physician 12 (1): 137-57.

[5] Slipman, C. W., Lipetz, J. S., Jackson, H. B., Rogers, D. P., and Vresilovic, E. J. 2000. "Therapeutic Selective Nerve Root Block in the Nonsurgical Treatment of Atraumatic Cervical Spondylotic Radicular Pain: A Retrospective Analysis with Independent Clinical Review." Archives of Physical Medicine and Rehabilitation 81 (6): 741-6.

[6] Anderberg, L., Anenrtz, M., Persson, L., Brandt, L., and Säveland, H. 2007. "Transforaminal Steroid Injection for the Treatment of Cervical Radiculopathy: A Prospective and Randomized Study.” Eur. Spin J. 16: 321-8.

[7] Scanlon, G. C., Moeller-Bertram, T., Romanowsky, S. M., and Wallace, M. S. 2007. "Cervical Transforaminal Epidural Steroid Injections: More Dangerous than We Think?" Spine 32: 1249-56.

[8] Razzaq, A. A., O'brien, D., Mattew, B., Bartlett, R., and Taylor, D. 2007. "Efficacy and Durability of Fluoroscopically Guided Cervical Nerve Root Block.” $\mathrm{Br}$. J. neurosurg 12: 365-9.

[9] Sampath, P., bendebba, M., Davis, J. D., and Ducker, T. 1999. "Outcome in Patients with Cervical Radiculopathy: Prospective, Multicenter Study with Independent Clinical Review." Spine 24: 591-7.
[10] Engquist M., Löfgren, H., Öberg, B., Holtz, A., Peolsson, A., Söderlund, A., Vavruch, L., and Lind, B. 2013. "Surgery versus Nonsurgical Treatment of Cervical Radiculopathy.” Spine 38 (20): 1715-22.

[11] Tanaka, Y., Kokubun, S., Sato, T., and Ozawa, H. 2006. "Cervical Roots as Origin of Pain in the Neck or Scapular Regions.” Spine 31 (17): e568-73.

[12] Jeon, T. S., Chang, H., and Choi, B. W. 2011. "Current Concept on the Operative Treatment for Degenerative Cervical Disc Disease." J. Korean Med. Assoc. 54: 941-50.

[13] Saal, J. S., Saal, J. A., and Yurth, E. F. 1996. "Nonoperative Management of Herniated Cervical Intervertebral Disc with Radiculopathy." Spine 21: 1877-83.

[14] Rathmell, J. P., Aprill, C., and Bogduk, N. 2004. "Cervical Transforaminal Injection of Steroids." Anesthesiology 100 (6): 1595-600.

[15] Radhakrishnan, K., Litchy, W. J., O'Fallon, W. M., and Kurland, L. T. 1994. "Epidemiology of Cervical Radiculopathy a Population-Based Study from Rochester, Minnesota, 1976 through 1990.” Brain 117 (2): 325-35.

[16] Engquist, M., Löfgren, H., Öberg, B., Holtz, A., Peolsson, A., Söderlund, A., Vavruch, L., and Lind, B. 2013. "Surgery versus Nonsurgical Treatment of Cervical Radiculopathy: A Prospective, Randomized Study Comparing Surgery plus Physiotherapy with Physiotherapy Alone with a 2-Year Follow-Up.” Spine 38 (20): 1715-22.

[17] Eck, J. C., Humphreys, S. C., Lim, T. H., Jeong, S. T., Kim, J. G., Hodges, S. D., and An, H. S. 2002. "Biomechanical Study on the Effect of Cervical Spine Fusion on Adjacent-Level Intradiscal Pressure and Segmental Motion." Spine 27 (22): 2431-4.

[18] Lee, G. M., Lee, E. Y., Han, J. H., Cho, K.H., Kang, S. R., and Yoon, S. H. 2014 "Effects of Wonli Acupuncture Procedure in Patients with LSS : A Clinical, Retrospective Study." Evidence-Based Complementary and Alternative Medicine 2014: 212098. 